\title{
Centrosomal Abnormalities in Pancreatic Cancer: Molecular Mechanisms and Clinical Implications
}

\author{
DANIEL ANSARI, CAROLINA DEL PINO BELLIDO, MONIKA BAUDEN and ROLAND ANDERSSON \\ Department of Surgery, Clinical Sciences Lund, Lund University, Skåne University Hospital, Lund, Sweden
}

\begin{abstract}
The centrosome is the main microtubuleorganizing center in human cells. It regulates normal cellcycle progression and cell division. Aberrations in the number, structure and function of centrosomes have been found to drive genomic instability and tumorigenesis. Pancreatic cancer frequently displays centrosomal aberrations. Supernumerary and abnormal centrosomes are observed in the earliest stages of pancreatic tumor development, and the p53 pathway acts as an initial barrier to the proliferation of cells with extra centrosomes. In this review, we summarize recent advances in the understanding of centrosomal aberrations in pancreatic cancer, focusing on regulatory mechanisms and prospects for future anticancer treatment.
\end{abstract}

The centrosome is an organelle that was discovered by Edouard van Beneden in 1883. The term 'centrosome' was coined in 1888 by Theodor Boveri. Centrosomes are the main microtubule-organizing centers in human cells. They are responsible for cell polarity and balanced chromosome segregation during mitosis in dividing cells. Increased numbers of centrosomes can give rise to multipolar spindles in mitosis, and thereby lead to aneuploidy and chromosomal instability. Centrosomal amplification has been associated with the initiation and progression of multiple malignancies (1-3). Furthermore, it has been reported that centrosomal amplification may be related to more aggressive tumor biology $(4,5)$.

This article is freely accessible online.

Correspondence to: Professor Roland Andersson, MD, Ph.D., Department of Surgery, Clinical Sciences Lund, Lund University and Skåne University Hospital, Lund, SE-221 85 Lund, Sweden. Tel: +46 46172359, e-mail: roland.andersson@med.lu.se

Key Words: Centrosomal abnormalities, pancreatic cancer, molecular mechanisms, treatment, review.
Pancreatic adenocarcinoma is one of the most severe types of cancer. The median survival is only 6 months after diagnosis and fewer than $5 \%$ of patients survive beyond 5 years (6). The poor outcome is attributed primarily to the lack of early diagnosis, as most patients are diagnosed with locally advanced or metastatic disease, when the chance for cure is minimal. Furthermore, pancreatic tumors are characterized by a high degree of resistance to conventional chemotherapy and radiation. Although much research has been directed towards understanding the molecular mechanisms behind pancreatic cancer, still little is known regarding the drivers of tumor initiation and progression.

Pancreatic tumors frequently exhibit centrosomal amplification and clustering, even in the earliest stages of tumor development (7). While centrosomal alterations have been linked to pancreatic cancer, it is still unclear whether these centrosomal abnormalities are a cause or a consequence of the disease. This review, discusses the potential underlying mechanisms for centrosomal abnormalities in pancreatic cancer and how they may be targeted for therapeutic gain.

\section{Centrosomal Amplification}

Centrosomal amplification is frequently detected in human cancer, mostly involved in the transition from early to advanced tumor stages, but also being found in some preneoplastic lesions. The presence of numeral alterations of centrosomes may lead to chromosomal instability and aneuploidy, increasing the risk for initiation of malignant transformation, tumor progression, chemoresistance and poor prognosis (1). Normal centrosomes form mitotic spindles that aid in proper chromosomal segregation in order to form stabilized daughter cells with the correct amount of DNA in each cell. Structural or functional abnormalities occurring in centrosomes may lead to chromosomal mis-segregation with subsequent aneuploidy and failure of cytokinesis. However, an excess of centrosomes does not necessarily lead to fatal 
cell division errors, but instead, may promote multipolar mitoses resulting in viable genomically unstable cells with phenotypic heterogeneity $(1,3,8,9)$.

Centrosomal amplification is predominantly found in pancreatic adenomas and carcinomas. Pancreatic tumors with centrosomal amplification are associated with worse clinical outcomes, including rapid disease progression and metastasis $(5,8,10)$. Amplified centrosomes enhance motility and invasiveness of pancreatic cancer cells, also contributing to intratumoral heterogeneity (5).

Centrosomal amplifications are, however, not involved in all types of pancreatic cancer in the same manner. Neuroendocrine pancreatic tumors lack such alterations in their pathogenesis. These tumor cells are usually welldifferentiated with few or no areas of atypia and display no loss of cellular polarity $(1,7)$. Thus, it may be speculated that centrosomal amplifications are partly responsible for the different pathology of these two kinds of tumors.

p53 involvement. More recently, there have been deeper investigations into centrosomal abnormalities and their link to oncogenic and tumor-suppressor proteins. The importance of p53 for centrosome activity and cell-cycle regulation must be emphasized. p53 is described as the 'guardian of the genome', because it conserves stability by preventing genomic mutation. In the case of DNA damage, p53 initiates cell-growth arrest or apoptosis in order to stop the proliferation of altered cells. Alterations in p53 lead to failure of cytokinesis, cells avoid the tetraploid checkpoint, resulting in pleiotropic defects. Consequently, there is also a rapid exacerbation of centrosomal amplification and aneuploidy due to unequal segregation $(1,7,11,12)$. Mutations in $p 53$ have been described in $75 \%$ of pancreatic cancer cases, and have been shown to lead to centrosomal amplification and numerical and structural chromosomal instability. Mutant p53 promotes the development of invasive and metastatic pancreatic ductal adenocarcinoma from pre-invasive disease. Loss of p53 regulation acts with oncogenic KRAS to drive chromosomal instability and tumor progression (13).

The role of kinases, such as polo-like kinase 4 (PLK4), is also important. PLK4 is a kinase that controls centrosomal duplication during mitosis. Its overexpression promotes pancreatic tumor formation (14). The absence of p53 allows altered cells with centrosomal amplification to undergo continuous division, leading to a neoplastic state. Induction of Plk4 in p53 null mice induces tumor formation with hyperproliferation in the pancreas. Such cells have multiple and abnormal centrosomes due to the overexpression of PLK4, and the absence of $\mathrm{p} 53$, which is a key in pancreatic tumorigenesis (15).

It has been suggested that centrosomal abnormalities may be sufficient to drive tumorigenesis in multiple tissues (16). The presence of extra centrosomes has been found to activate the p53-induced protein with a death domain (PIDDosome), a multiprotein complex, leading to caspase-2-mediated cleavage of murine double minute-2 (MDM2) and p53 accumulation (16).

CEP70 involvement. Other mechanisms involve centrosomal protein of $70 \mathrm{kDa}$ (CEP70). This protein is located in the centrosome throughout the cell cycle and interacts with gamma tubulin. CEP70 has a critical role in regulation of microtubule dynamics, mitotic spindle formation, cell migration and ciliogenesis and its overexpression results in centrosome abnormality $(17,18)$. CEP70 is overexpressed in pancreatic cancer and has an important correlation with centrosomal amplification and clinical parameters (e.g. histological grade, TNM stage) (11).

\section{Centrosomal Regulation}

Further support for the concept that different proteins and routes affect centrosomal regulation, leading to pancreatic cancer, comes from experimental studies carried out on the pathways controlling the cell cycle. Some of these pathways involve proteins such as protein phosphatase 4 (PP4) or CEP55 that are clearly related to centrosomal maturation and microtubule growth. PP4 has been found to be overexpressed in pancreatic cancer and is considered as a prognostic factor in patients with pancreatic cancer. It plays an important role in the organization of the centrosome, as well as its maturation in mitosis and meiosis (19). CEP55 functions as a microtubule-binding protein that participates in cell mitosis. CEP55 is overexpressed in pancreatic cancer, promoting the growth and invasion of cancer cells (20). Factors connected to coordination of the cell cycle play an important role in centrosomal regulation. Any alteration in this process may lead to centrosomal abnormalities and consequently initiate pancreatic cancer (21-24).

Another important group of kinases that contribute to the correct functioning of centrosomes is the Aurora family. Aurora serine/threonine kinases regulate chromosomal segregation and cytokinesis, and are necessary for completing mitotic events such as centrosomal separation and bipolar spindle assembly. The role of Aurora kinases in pancreatic cancer evolution and their relation to centrosomal abnormalities has been widely studied. It has been shown that abnormal Aurora expression induces transition of altered pancreatic cells into the cell cycle, and leads to centrosomal amplification and chromosomal instability. The affected cells survive apoptosis and their aneuploidy drives tumor progression by enhancing genomic instability, which results in massive alterations of cellular phenotypes (25-27). There is a strong correlation between the degree of chromosomal instability in cells with altered AURORA kinases and pancreatic tumor behavior. Pancreatic cells possessing minimal deviations in the chromosome copy number were found to be clinically less aggressive than those with major 


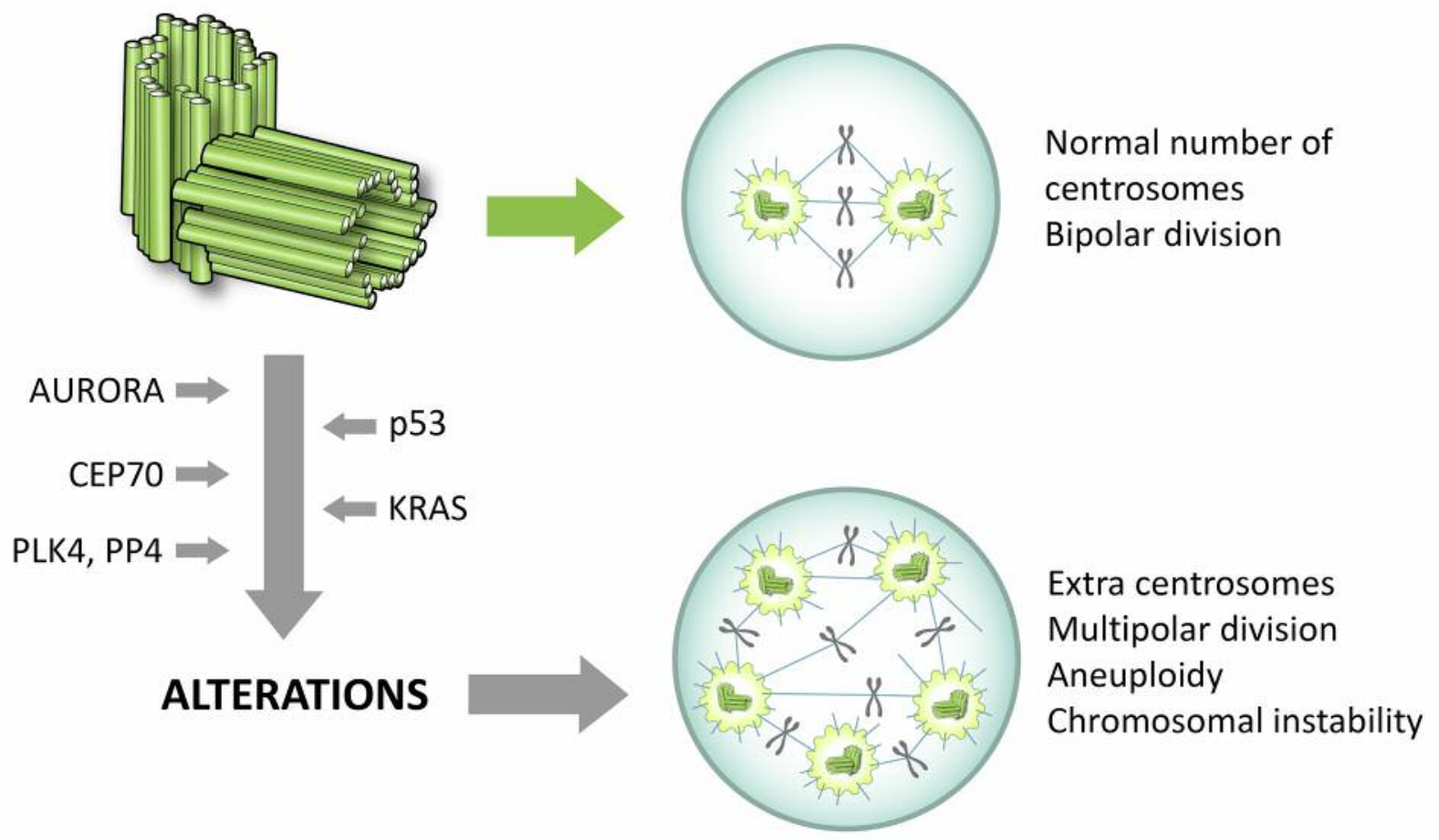

Figure 1. The role of centrosomes in pancreatic cancer development and progression. CEP70: Centrosomal protein of 70 kDa; PLK4: polo-like kinase 4; PP4: protein phosphatase 4.

increases in their total nuclear DNA content. These alterations are clearly due to the defects in the mitotic spindles and in the centrosomes, caused by mutation of kinases (28-30).

\section{Diagnosis and Treatment}

Centrosomal amplification and clustering occur in the early stages of tumorigenesis. Identification of these abnormalities would be attractive for early detection of pancreatic cancer. However, the concept of using centrosomal alterations as diagnostic biomarkers for pancreatic cancer is not well established and there is no relevant literature in this area as far as we are aware.

On the other hand, the concept of centrosomes as therapeutic targets is more developed. Centrosome declustering drugs (such as antifungals) disaggregate the centrosome clusters and force the mitotic spindle to adopt a persistently high grade multipolar configuration, which is incompatible with cell survival. In pancreatic cancer, there are pseudobipolar spindle configurations where these drugs could be useful (5).

Pancreatic cancer is known not only for its aggressiveness, but also for its lack of response to chemotherapy. The Aurora kinase family have an important role in this process, and it has been found that specific knockdown of these kinases in cultured pancreatic cancer cells strongly suppressed cell growth and tumorigenesis by inducing apoptosis. When used in combination with taxanes, pancreatic cancer cell chemosensitivity was enhanced (31). Other studies also reported that inhibition of Aurora kinases is useful for inducing chemosensitivity and leads to cell-cycle arrest, making them a potential target for drug development $(9,26,27)$.

Other treatment approaches include radiation and serum depletion. Radiation induces death in solid tumors normally by mitotic cell death. The irradiation causes centrosomal overduplication and multipolar spindles. Irradiated pancreatic cancer cells treated with serum depletion exhibited an increase in the number of centrosomal abnormalities, which promoted nuclear fragmentation and cell death (32).

\section{Conclusion}

Centrosomal amplification has been found to cause chromosomal instability and aneuploidy, contributing to pancreatic tumor development (Figure 1). Centrosomal amplification is likely an early event in pancreatic tumorigenesis and also correlates with an aggressive disease phenotype. The p53 pathway appears to be compromised in pancreatic tumors that arise following centrosomal amplification. Cancer cells, unlike normal cells, rely on centrosome clustering for survival. In the future, analysis of intratumoral centrosomal aberrations may aid in risk 
stratifying patients with pancreatic tumors, and tumorspecific treatment may be possible through use of centrosome declustering drugs or substances that target centrosomal proteins (e.g. PLKs, Aurora kinase family).

\section{Funding}

The study was supported by funding from SWElife/Vinnova, the Royal Physiographic Society of Lund, the Magnus Bergvall Foundation, the Tore Nilsson Foundation and the Inga and John Hain Foundation for Medical Research.

\section{References}

1 Chan JY: A clinical overview of centrosome amplification in human cancers. Int J Biol Sci 7: 1122-1144, 2011.

2 Gonczy P: Centrosomes and cancer: revisiting a long-standing relationship. Nat Rev Cancer 15: 639-652, 2015.

3 Godinho SA and Pellman D: Causes and consequences of centrosome abnormalities in cancer. Philos Trans R Soc Lond B Biol Sci 369: 20130467, 2014.

4 Pannu V, Mittal K, Cantuaria G, Reid MD, Li X, Donthamsetty S, McBride M, Klimov S, Osan R, Gupta MV, Rida PC and Aneja R: Rampant centrosome amplification underlies more aggressive disease course of triple negative breast cancers. Oncotarget 6: 10487-10497, 2015.

5 Mittal K, Ogden A, Reid MD, Rida PC, Varambally S and Aneja $\mathrm{R}$ : Amplified centrosomes may underlie aggressive disease course in pancreatic ductal adenocarcinoma. Cell Cycle 14: 2798-2809, 2015.

6 Ansari D, Tingstedt B, Andersson B, Holmquist F, Sturesson C, Williamsson C, Sasor A, Borg D, Bauden M and Andersson R: Pancreatic cancer: yesterday, today and tomorrow. Future Oncol 12: 1929-1946, 2016.

7 Sato N, Mizumoto K, Nakamura M, Nakamura K, Kusumoto M, Niiyama H, Ogawa T and Tanaka M: Centrosome abnormalities in pancreatic ductal carcinoma. Clin Cancer Res 5: 963-970, 1999.

8 Sato N, Mizumoto K, Nakamura M, Maehara N, Minamishima YA, Nishio S, Nagai E and Tanaka M: Correlation between centrosome abnormalities and chromosomal instability in human pancreatic cancer cells. Cancer Genet Cytogenet 126: 13-19, 2001.

9 Difilippantonio MJ, Ghadimi BM, Howard T, Camps J, Nguyen QT, Ferris DK, Sackett DL and Ried T: Nucleation capacity and presence of centrioles define a distinct category of centrosome abnormalities that induces multipolar mitoses in cancer cells. Environ Mol Mutagen 50: 672-696, 2009.

10 Shono M, Sato N, Mizumoto K, Maehara N, Nakamura M, Nagai E and Tanaka M: Stepwise progression of centrosome defects associated with local tumor growth and metastatic process of human pancreatic carcinoma cells transplanted orthotopically into nude mice. Lab Invest 81: 945-952, 2001.

11 Xie S, Qin J, Liu S, Zhang Y, Wang J, Shi X, Li D, Zhou J and Liu M: Cep70 overexpression stimulates pancreatic cancer by inducing centrosome abnormality and microtubule disorganization. Sci Rep 6: 21263, 2016.

12 Mizumoto K and Tanaka M: Genetic diagnosis of pancreatic cancer. J Hepatobiliary Pancreat Surg 9: 39-44, 2002.
13 Hingorani SR, Wang L, Multani AS, Combs C, Deramaudt TB, Hruban RH, Rustgi AK, Chang $\mathrm{S}$ and Tuveson DA: Trp53R172H and KrasG12D cooperate to promote chromosomal instability and widely metastatic pancreatic ductal adenocarcinoma in mice. Cancer Cell 7: 469-483, 2005.

14 Coelho PA, Bury L, Shahbazi MN, Liakath-Ali K, Tate PH, Wormald S, Hindley CJ, Huch M, Archer J, Skarnes WC, ZernickaGoetz $\mathrm{M}$ and Glover DM: Overexpression of Plk4 induces centrosome amplification, loss of primary cilia and associated tissue hyperplasia in the mouse. Open Biol 5: 150209, 2015.

15 Levine DS, Sanchez CA, Rabinovitch PS and Reid BJ: Formation of the tetraploid intermediate is associated with the development of cells with more than four centrioles in the elastase-simian virus 40 tumor antigen transgenic mouse model of pancreatic cancer. Proc Natl Acad Sci USA 88: 6427-6431, 1991.

16 Fava LL, Schuler F, Sladky V, Haschka MD, Soratroi C, Eiterer L, Demetz E, Weiss G, Geley S, Nigg EA and Villunger A: The PIDDosome activates $\mathrm{p} 53$ in response to supernumerary centrosomes. Genes Dev 31: 34-45, 2017.

17 Shi X, Liu M, Li D, Wang J, Aneja R and Zhou J: Cep70 contributes to angiogenesis by modulating microtubule rearrangement and stimulating cell polarization and migration. Cell Cycle 11: 1554-1563, 2012.

18 Yang Y, Ran J, Liu M, Li D, Li Y, Shi X, Meng D, Pan J, Ou G, Aneja R, Sun SC and Zhou J: CYLD mediates ciliogenesis in multiple organs by deubiquitinating CEP70 and inactivating HDAC6. Cell Res 24: 1342-1353, 2014.

19 Weng S, Wang H, Chen W, Katz MH, Chatterjee D, Lee JE, Pisters PW, Gomez HF, Abbruzzese JL, Fleming JB and Wang H: Overexpression of protein phosphatase 4 correlates with poor prognosis in patients with stage II pancreatic ductal adenocarcinoma. Cancer Epidemiol Biomarkers Prev 21: 1336-1343, 2012.

20 Peng T, Zhou W, Guo F, Wu HS, Wang CY, Wang L and Yang ZY: Centrosomal protein 55 activates NF-kappaB signalling and promotes pancreatic cancer cells aggressiveness. Sci Rep 7: 5925, 2017.

21 Ning Z, Wang A, Liang J, Liu J, Zhou T, Yan Q and Wang Z: Abnormal expression of NEK2 in pancreatic ductal adenocarcinoma: a novel marker for prognosis. Int J Clin Exp Pathol 7: 2462-2469, 2014.

22 Kim JJ, Rajagopalan K, Hussain B, Williams BH, Kulkarni P and Mooney SM: CETN1 is a cancer testis antigen with expression in prostate and pancreatic cancers. Biomark Res 1: 22, 2013.

23 Jia J, Bosley AD, Thompson A, Hoskins JW, Cheuk A, Collins I, Parikh H, Xiao Z, Ylaya K, Dzyadyk M, Cozen W, Hernandez BY, Lynch CF, Loncarek J, Altekruse SF, Zhang L, Westlake CJ, Factor VM, Thorgeirsson S, Bamlet WR, Hewitt SM, Petersen GM, Andresson T and Amundadottir LT: CLPTM1L promotes growth and enhances aneuploidy in pancreatic cancer cells. Cancer Res 74: 2785-2795, 2014.

24 Giamas G, Hirner H, Shoshiashvili L, Grothey A, Gessert S, Kuhl M, Henne-Bruns D, Vorgias CE and Knippschild U: Phosphorylation of CK1delta: identification of Ser370 as the major phosphorylation site targeted by PKA in vitro and in vivo. Biochem J 406: 389-398, 2007.

25 Zhu J, Abbruzzese JL, Izzo J, Hittelman WN and Li D: AURKA amplification, chromosome instability, and centrosome abnormality in human pancreatic carcinoma cells. Cancer Genet Cytogenet 159: 10-17, 2005. 
26 Chen J, Li D, Wei C, Sen S, Killary AM, Amos CI, Evans DB, Abbruzzese JL and Frazier ML: Aurora-A and p16 polymorphisms contribute to an earlier age at diagnosis of pancreatic cancer in Caucasians. Clin Cancer Res 13: 31003104, 2007.

27 Rojanala S, Han H, Munoz RM, Browne W, Nagle R, Von Hoff DD and Bearss DJ: The mitotic serine threonine kinase, aurora2 , is a potential target for drug development in human pancreatic cancer. Mol Cancer Ther 3: 451-457, 2004.

28 Li D, Zhu J, Firozi PF, Abbruzzese JL, Evans DB, Cleary K, Friess $H$ and Sen $S$ : Overexpression of oncogenic STK15/BTAK/aurora A kinase in human pancreatic cancer. Clin Cancer Res 9: 991-997, 2003.

29 Barr AR and Gergely F: Aurora-A: the maker and breaker of spindle poles. J Cell Sci 120: 2987-2996, 2007.

30 Kobayashi $\mathrm{T}$ and Dynlacht BD: Regulating the transition from centriole to basal body. J Cell Biol 193: 435-444, 2011.
31 Hata T, Furukawa T, Sunamura M, Egawa S, Motoi F, Ohmura $\mathrm{N}$, Marumoto T, Saya $\mathrm{H}$ and Horii A: RNA interference targeting aurora kinase a suppresses tumor growth and enhances the taxane chemosensitivity in human pancreatic cancer cells. Cancer Res 65: 2899-2905, 2005.

32 Shono M, Sato N, Mizumoto K, Minamishima YA, Nakamura M, Maehara N, Urashima T, Saimura M, Qian L, Nishio S, Nagai E and Tanaka M: Effect of serum depletion on centrosome overduplication and death of human pancreatic cancer cells after exposure to radiation. Cancer Lett 170: 81-89, 2001.

Received December 19, 2017

Revised January 18, 2018

Accepted January 24, 2018 